\title{
EEG feature extraction for imagery movement based on improved WICA method
}

\author{
Nianqiang Li Yongling Wang \\ Shandong Provincial Key Laboratory of Network Based Intelligent Computing; \\ School of Information Science and Engineering; University of Jinan
}

\begin{abstract}
$\mathrm{Mu}$ rhythm is produced in motor sensory cortex of human brain, a region associated with voluntary movement. Feature extraction for imagery movement related to in this paper is based on mu rhythm. The result of the new method we put forward showed that we can separate EEG signal from power frequency noise. We extracted the mu rhythm by wavelet transform, enhancing features using improved ICA algorithm. We successfully extracted the mu rhythm feature with improved WICA and the experiment results demonstrate that the new method is effective.
\end{abstract}

Keywords: Mu rhythm; imagery movement; feature extraction; improved WICA

\section{Introduction}

Motor imaginary potentials refers to the scalp potential changes in the motor cortex area of the brain evoked neural electrical activity caused.The experiment of this paper is based on controlling the mu rhythm by single action imagine task. When we collect EEG data through EEG acquisition equipment, it will mix with AC interference. ICA method ${ }^{[1]}$ application can separate EEG signal from interference signal effectively in the sense of statistical independence. This paper we will combine extended informax algorithm and fast ICA algorithm to separate the EEG signal mixed with AC interference. The experimental results show that ICA method can effectively remove these artifacts.

Wavelet transform ${ }^{[2]}$ can enhance the useful information meanwhile weaken the off-target signal components. As wavelet transform just extracts frequency band we interested in, we use fast ICA to separate mu rhythm from EEG signal. The results of the experiment showed that ICA can separate the relatively strong target component effectively.

\section{Materials and methods}

\subsection{Data Collection}

EEG data was from one healthy partner of our laboratory. The subject is required completely relax. From one to twenty seconds, the subject was relax and tried to think nothing. From twenty to thirty seconds the subject did his best to think the action "drinking", just repeat. Electrodes were placed at the following 16 locations: Fp1, F3, C3, P3, O1, F7, T3, T5, Fp2, F4, $\mathrm{C} 4, \mathrm{P} 4, \mathrm{O} 2, \mathrm{~F} 8, \mathrm{~T} 4$ and $\mathrm{T} 6$ with a reference at FCz. The EEG was recorded at least $1 \mathrm{~min}$. we chose $\mathrm{C} 4$ channel which corresponds to motor center of human brain. We select 23 to 27 seconds as the research object, in which time the signal is stable and the features are showed obviously as Figure. 1 shows. EEG data was digitally recorded at a rate of 1000 sam- 
ples/s using EEG acquisition equipment of our laboratory.

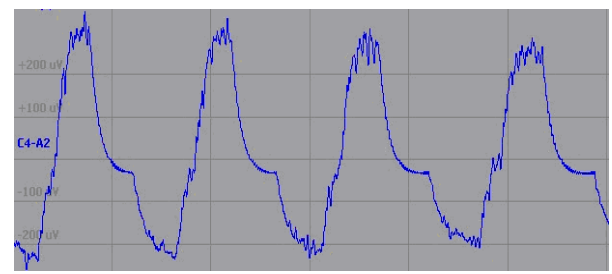

Fig.1: C4-A2 channel source signal from 23 to 27 seconds.

\subsection{Independent Component Analysis}

Independent Component Analysis (ICA) aims at blindly decomposing a linear mixture of independent sources. The ICA model of a linear instantaneous mixture can be formulated as

$$
\mathrm{x}=A s
$$

where $s=\left[s_{1}, \ldots, s_{m}\right]^{T}$ is a vector of independent sources. These independent sources are mixed through a linear system $A$. In this paper, we used two important ICA algorithms ${ }^{[3]}$.

\subsubsection{Extended informax ICA algo- rithm}

The traditional infomax algorithm is a blind source separation algorithm based on neural network information maximum transmission guidelines ${ }^{[4]}$. The corresponding learning rule as below,

$$
\Delta W=\eta\left[I-2 \tanh (y) y^{T}\right] W
$$

To separate signals including superGaussian and sub-Gaussian, the unmixing matrix of extended informax adjustment formula is:

$$
\Delta W=\eta\left[I-\operatorname{sign}(k 4) \tanh (y) y^{T}-y y^{T}\right] W
$$

Using extended informax ICA algorithm $^{[5]}$ to process EEG signal can come out online learning but with slow convergence rate based on linear convergence.

\subsubsection{Fast ICA algorithm}

Fixed point of independent component analysis algorithm ${ }^{[6]}$ separates the independent components by measuring the non-Gaussianity through the value of negentropy. The definition of negentropy is

$$
J(\mathrm{y})=H\left(y_{\text {gauss }}\right)-H(y)
$$

The Shannon entropy function denoted by $H($.$) is defined as$

$$
H(Y)=-\int p_{Y}(\xi) \lg p_{Y}(\xi) d \xi
$$

Fast ICA algorithm iterative formula is,

$$
\begin{gathered}
W_{i}(k+1)=E\left[z f\left(W_{i}^{T}(k) z\right)\right]-E\left[f^{\prime}\left(W_{i}^{T}(k) z\right)\right] W_{i}(k) \\
W_{i}(k+1) \leftarrow \frac{W_{i}(k+1)}{\left\|W_{i}(k+1)\right\|_{2}}
\end{gathered}
$$

Using fast ICA algorithm to process EEG signal can realize rapid convergence but could not come out online learning.

\subsubsection{Improved informax-fast algo- rithm}

Informax algorithm can realize online learning but has slow convergence speed. Instead fast ICA algorithm has fast convergent rate but could not realize online learning. Procedure is as follows: Removing mean and whitening. Choose the number of the independent components we need to estimate. Loop several steps to get initial value $\mathrm{W}$ by extended informax algorithm. W isn't required converge. Take the $\mathrm{W}$ we get as the initial value $\mathrm{W}$ in fast ICA algorithm. Run by fast ICA until it converge.

With improved ICA algorithm, we processed EEG signal with rapid convergence and online learning applying to denoising and feature extraction.

\subsection{Wavelet transform}

Discrete wavelet transform is defined as: 


$$
\begin{aligned}
& W_{f}(m, n)=\int_{R} f(t) \Psi_{m, n}^{\prime}(t) d t \\
& \Psi_{m, n}(t)=2^{-m / 2} \Psi\left(2^{-m} t-n b_{0}\right)
\end{aligned}
$$

As for discrete signal $\mathrm{f}(n)$, we can decomposed the signal with mallat algorithm $^{[7]}$.

$$
\mathrm{f}(n)={f_{L}}^{\mathrm{a}}(n)+\sum_{j=1}^{L}{f_{j}}^{d}(n)
$$

With wavelet transform we decomposed the EEG signal into 7 layers and extract initial mu rhythm with energy of the third frequency band as feature information.

\section{Preprocessing of the EEG signal}

EEG signal will be affected by AC interference more or less. In order to remove AC interference from EEG signal, we built a power frequency noise signal as

$$
s 1(t)=A \sin (2 \pi f t+n), f=1000
$$
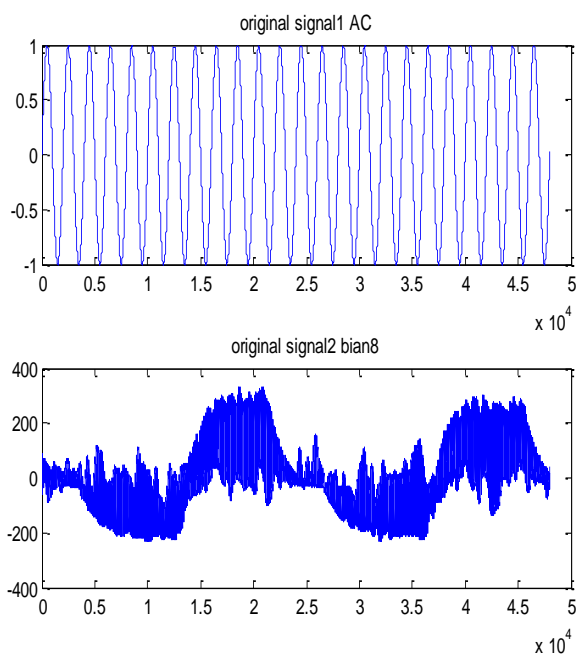

Fig. 2: EEG signal with AC interference.
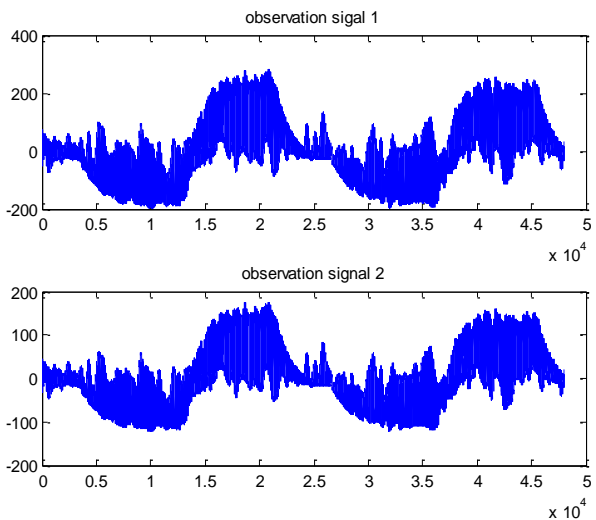

Fig. 3: mixed signal linear signal.
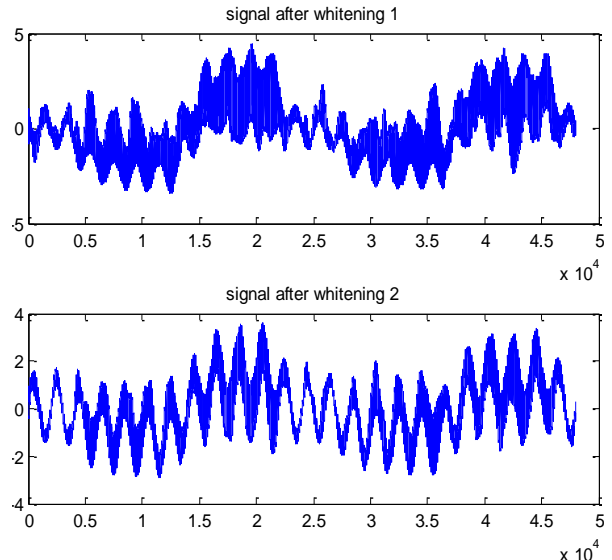

Fig. 4: signal after whitening.

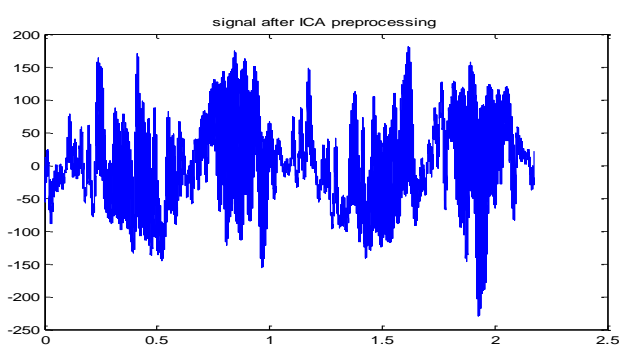

Fig. 5: EEG signal without $\mathrm{AC}$ in terference Figure.

We can see the signal after removing mean and whitening processing showed in Figure. 3 and Figure.4. At last we made the separate component of $\mathrm{AC}$ interference zero. Figure. 5 showed the EEG signal without $\mathrm{AC}$ interference. 


\section{EEG feature extracting with wave- let transform}

The preprocessing of the EEG signal is decomposed 7 levels by wavelet transform. We chose DB4 wave because the shape of the DB wave is the same with EEG rhythm. As shown in the Figure.6, we can see decomposition structure contains the coefficients of wavelet decomposition $\mathrm{C}$ and coefficient vector $\mathrm{L}$. The Figure.7 showed the coefficients of each level after wavelet decomposition.
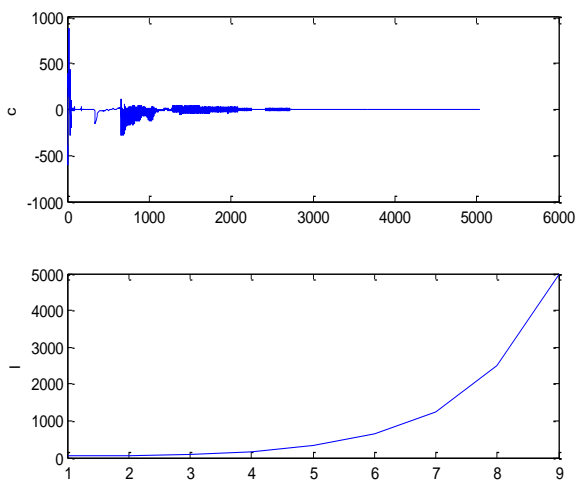

Fig. 6: the decomposition vector $\mathrm{C}$ and coefficient length.

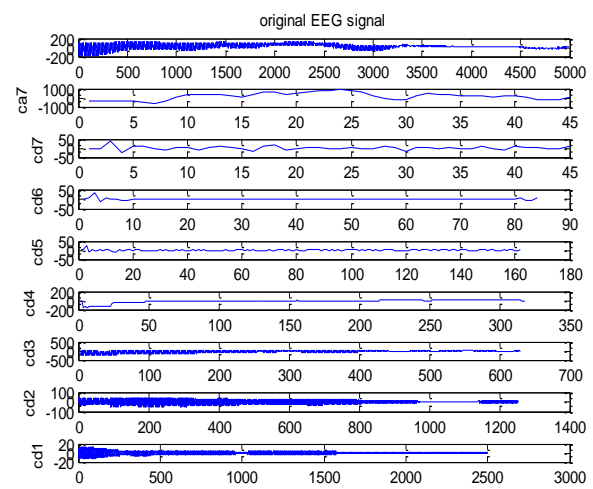

Fig. 7: the processing result of the wavelet decomposition.

Analysis of all the level of power spectral density is showed in Figure.8, from which we can conclude that the effect of the third level is the biggest. The first level is the power spectrum of original EEG signal.

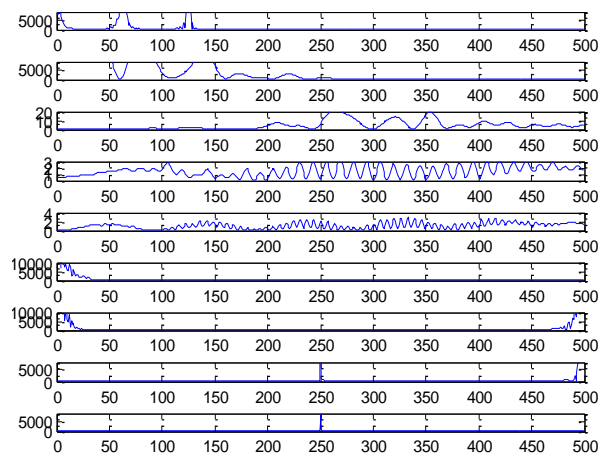

Fig. 8: power spectrum after analysis of WT.

We choose the third level processing result of wavelet construction whose energy is from $10 \sim 13 \mathrm{HZ}$ as the rhythm feature. Its power spectrum is showed as Figure.10.

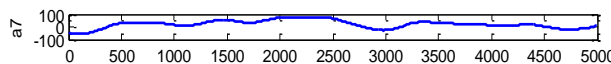
$50_{-5}^{5}$ $\begin{array}{lllllllllll}-50 & 500 & 1000 & 1500 & 2000 & 2500 & 3000 & 3500 & 4000 & 4500 & 5000\end{array}$

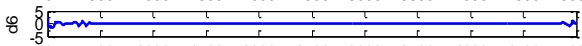
$\begin{array}{lllllllllll}-50 & 500 & 1000 & 1500 & 2000 & 2500 & 3000 & 3500 & 4000 & 4500 & 5000\end{array}$

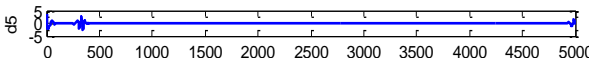
8.

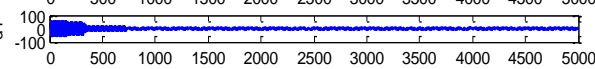

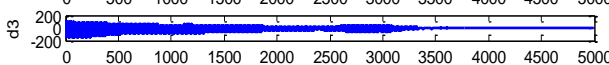

은 50

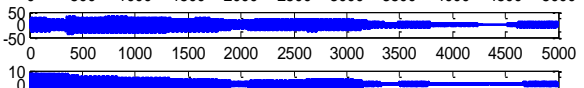

ᄃ 10

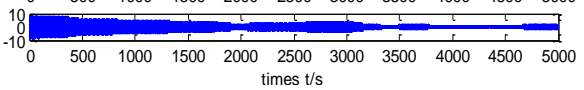

Fig. 9: processing result of wavelet construction.
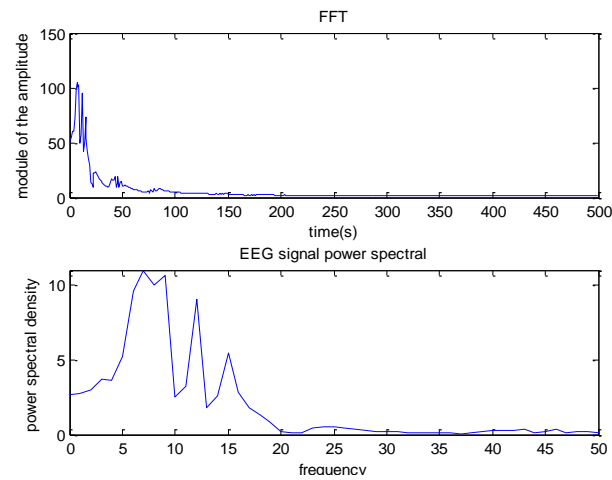

Fig. 10: the second level of power spectrum. 


\section{EEG feature extracting with fast ICA}

Taking the result of WT processing as the input of the fast ICA, we put the original signal after preprocessing as the fast ICA input.

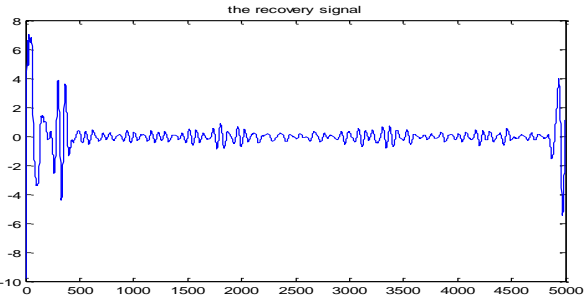

Fig. 11: Result of mu rhythm with improved ICA.

The signal with WT processing can be seen reference signal. Then we run it with fast ICA. The result by fast ICA running is showed in Figure.11.

\section{Analysis of the result}

We can compare the result with improved WICA method as shown in Figure.11 with methods included wavelet transform, ICA and traditional WICA method. We can see the result as shown in Figure.12, Figure.13, and Figure.14. By comparing the power spectrum of each method, we can see the frequency between 10-13HZ, the effect of the improved method simulating result is better than the other methods.
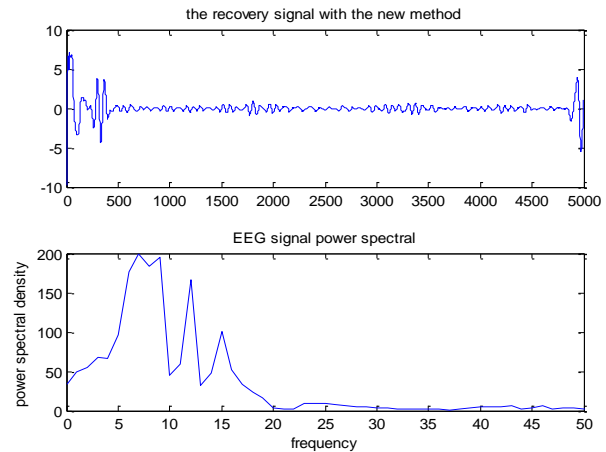

Fig. 12: Result with improved WICA and power spectrum .
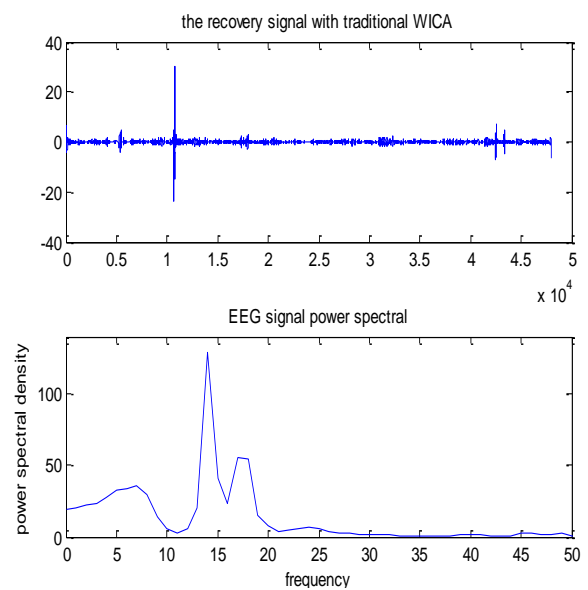

Fig. 13: Result with traditional WICA and power spectrum .
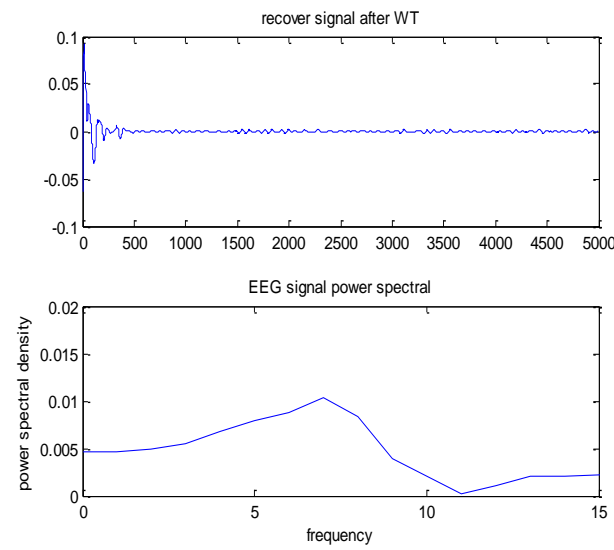

Fig. 14: Result with WT and power spectrum.
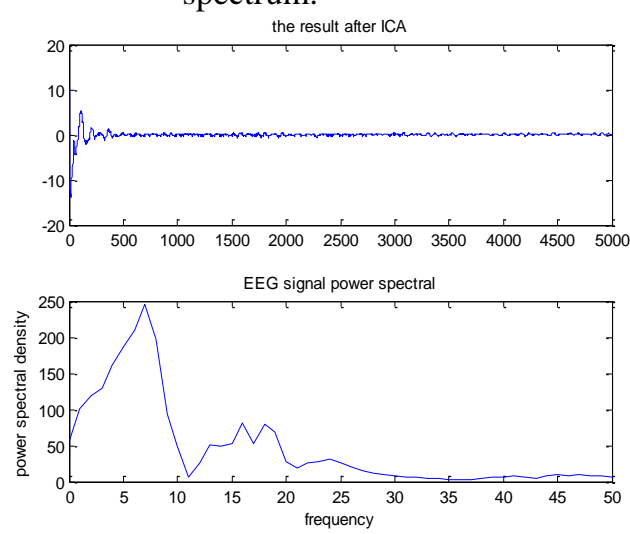

Fig. 15: Result with ICA and power spectrum. 


\section{Conclusions}

ICA has been an efficient tool for feature extraction. A new method the improved WICA is proposed in this study and the effectiveness of this method is demonstrated by applying it to EEG signals. Actually, it successfully extracted the mu rhythm which is hidden in the original signals. We can extract other useful information using this method effectively. The work we have done in this paper plays an important role for the next feature classification in the BCI.

\section{References}

[1] Co Melissant, Alexander Ypmaa, Edward E.E. Frietmana, and Cornelis J. Stamb, "A method for detection of Alzheimer's disease using ICAenhanced EEG measurements," Artificial Intelligence in Medicine, pp. 209-222, 2005.

[2] Wu Ting, Yan Guozheng, Yang Banghua, and Sun Hong, "EEG feature extraction based on wavelet packet decomposition for brain computer interface," Measurement, pp. 618-625, 2008.
[3] Zhou Weidong, Jean Gotman, "Automatic removal of eye movement artifacts from the EEG using ICA and the dipole model," Progress in Natural Science, pp. 1165-1170, 2009.

[4] Lou Shan, "Study on Critical Techniques of the Active Portable BrainComputer Interface System," Qinghua university, pp. 35-53, 2009.

[5] Maarten De Vos, Lieven De Lathauwer, and Sabine Van Huffel, "Spatially constrained ICA algorithm with an application in EEG processing," Signal Processing, pp. 1963-1972, 2011.

[6] Jing Sui, Tülay Adali, Godfrey D. Pearlson, and Vince D. Calhoun, "An ICA-based method for the identification of optimal FMRI features and components using combined group-discriminative techniques," Clinical Neurophysiology, pp. 73-86, 2009.

[7] Abdulhamit Subasi, "EEG signal classification using wavelet feature extraction and a mixture of expert model," Expert Systems with Applications, pp. 1084-1093, 2007. 